\title{
Global Existence and Blow-Up for the Pseudo-parabolic $p(x)$-Laplacian Equation with Logarithmic Nonlinearity
}

\section{Fugeng Zeng ${ }^{1}$ (1) $\cdot$ Qigang Deng $^{1} \cdot$ Dongxiu Wang $^{1}$}

Received: 19 July 2021 / Accepted: 19 September 2021 / Published online: 30 September 2021

(C) The Author(s) 2021

\section{Abstract}

In this paper, we study the initial boundary value problem of the pseudo-parabolic $p(x)$-Laplacian equation with logarithmic nonlinearity. The existence of the global solution is obtained by using the potential well method and the logarithmic inequality. In addition, the sufficient conditions of the blow-up are obtained by concavity method.

Keywords Pseudo-parabolic · Variable exponent - Global existence · Blow-up · Logarithmic nonlinearity

Mathematics Subject Classification $35 \mathrm{~K} 59 \cdot 35 \mathrm{~K} 55 \cdot 35 \mathrm{~B} 40$

\section{Introduction}

The main purpose of this paper is to study the existence and blow-up of solutions for the following pseudo-parabolic $p(x)$-Laplacian equation with logarithmic nonlinearity:

$$
\begin{cases}u_{t}-\Delta u_{t}-\Delta_{p(x)} u=|u|^{q(x)-2} u \log (|u|), & x \in \Omega, t>0, \\ u(x, t)=0, & x \in \partial \Omega, t>0, \\ u(x, 0)=u_{0}(x), & x \in \Omega,\end{cases}
$$

where $\Delta_{p(x)} u=\operatorname{div}\left(|\nabla u|^{p(x)-2} \nabla u\right)$ is the $p(x)$-Laplacian, $\Omega \subset \mathbb{R}^{N}(N \geq 1)$ is a bounded domain with smooth boundary $\partial \Omega, u_{0}: \Omega \rightarrow \mathbb{R}$ is the initial function, and $p, q: \bar{\Omega} \rightarrow \mathbb{R}_{+}$are continuous functions which satisfy the following conditions:

Fugeng Zeng

zengfugeng@foxmail.com

1 School of Date Science and Information Engineering, Guizhou Minzu University, Guiyang 550025, China 


$$
2<p_{-}<p(x)<p_{+}<q_{-}<q(x)<q_{+}<p^{*}(x), \forall x \in \bar{\Omega}
$$

with

$$
p^{*}(x):= \begin{cases}+\infty & \text { if } \mathrm{N} \leq p_{+}, \\ \frac{N p(x)}{N-p(x)} & \text { if } \mathrm{N}>p_{+} .\end{cases}
$$

Pseudo-parabolic equations are characterized by the occurrence of a time derivative appearing in the highest order term, which can be wildly used in some physical and biological scenarios, such as the seepage of homogeneous fluids through a fissured rock, the heat conduction involving two temperature systems, the unidirectional propagation of nonlinear, dispersive, long waves, fluid flow in fissured porous media, two phase flow in porous media with dynamical capillary pressure and the aggregation of populations (see [1, 2]). For problem (1), we can also give an example about the non-stationary process in semiconductors in the presence of sources, where $\Delta u$ represents the linear dissipation of free charge current, $\Delta u_{t}-u_{t}$ represents the free electron density rate and the nonlinear term including the $p(x)$-Laplacian and logarithmic nonlinearity stands for the source of free electron current (see [3]). In particular, the pseudo-parabolic equations involving the $p(x)$-Laplacian can be used to study electrorheological fluids which are characterized by their ability to change the mechanical properties under the influence of the external electromagnetic, more introductions on physical motivations can be found in $[4,5]$ and the references contained therein.

In the past years, many authors make efforts to the investigation of the existence and blow-up of solutions for such kinds of equations. In Chen et al. [6], investigated the following semilinear heat equation with logarithmic nonlinearity

$$
u_{t}-\Delta u=u \log (|u|),
$$

they obtained the existence and blow-up at $+\infty$ of solutions of problem (2), they further proved that the global weak solution decayed exponentially in the case of $p=2$, and decayed algebraically in the case of $p>2$. Subsequently, Peng and Zhou [7] considered the following initial boundary value problem of semilinear heat equation with logarithmic nonlinearity

$$
\frac{\partial u}{\partial t}-\Delta u=|u|^{p-2} u \log |u|
$$

where $p$ satisfies

$$
2<p<2^{*}:=\left\{\begin{array}{l}
+\infty \text { if } n \leq 2 \\
\frac{2 n}{n-2} \text { if } n \geq 3
\end{array}\right.
$$

they obtained the existence of global solution, finite time blow-up and the upper bound of blow-up time of problem (3). For problem

$$
u_{t}-\Delta u_{t}-\Delta_{p} u=\varphi_{p}(u) \log (|u|),
$$


where $\varphi_{p}(z)=|z|^{p-2} z$. In case of $2<p<2^{*}$, the existence or nonexistence of global weak solutions as well as finite time blow-up phenomenon were obtained in [8]. In He et al. [9], considered the following nonlinear equation

$$
u_{t}-\Delta u_{t}-\Delta_{p} u=|u|^{q-2} u \log (|u|)
$$

they obtained result of decay estimation and finite blow-up of solutions where $2<p<q<p\left(1+\frac{2}{n}\right)$. Recently, Boudjeriou [10] considered the following nonlinear equation

$$
u_{t}-\Delta_{p(x)} u=|u|^{s(x)-2} u \log (|u|)
$$

which involved the $p(x)$-Laplacian operator with nonlinearities of variable exponent type. They proved that the local solutions of problem (6) blew up in finite time under suitable conditions. More results about partial differential equations involving the $p(x)$-Laplacian operator, we refer to [11-14] and the references therein.

A powerful technique for solving the existence of the above problem is the so-called potential well method, which was established by Tsutsumi [15], Levine [16], Payne and Sattinger in [17]. Liu et al. [18, 19] generalized and improved the method by introducing a family of potential wells which include the known potential well as a special case. On the other hand, the physical interpretation of blow-up phenomena is generally thought of as a dramatic increase in temperature which leads to ignition of a chemical reaction. Many researchers applied different methods to derive the sufficient conditions for finite time and infinite time blowup result. In particular, Levine [16] studied the abstract equation

$$
p \frac{d u}{d t}=-A(t) u+f(u(t)), t \in[0,+\infty)
$$

where $p$ and $A$ are positive linear operators defined on a dense subdomain $D$ of a real or complex Hilbert Space, in which they obtained the blow-up solutions, under abstract conditions

$$
2(\alpha+1) F(x) \leq(x, f(x)), F\left(u_{0}(x)\right)>\frac{1}{2}\left(u_{0}(x), A u_{0}(x)\right)
$$

for every $x \in D$, where $F(x)=\int_{0}^{1}(f(\rho x), x) d \rho$. This work has been recognized as a creative and elegant tool for giving criteria for the blow-up, which is called the concavity method. Nowadays, it is one of the most useful method for blow-up of solutions for evolution equations.

Inspired by the above works, by using the potential well method and concavity method, we consider the existence and blow-up of solutions for problem (1) which combine with pseudo-parabolic, variable exponent and logarithmic nonlinearity term. To our best knowledge, it is the first attempt to study the properties of the solutions for such kind of equations.

Here we give some important definitions as follows: for $u_{0} \in W_{0}^{1, p(x)}(\Omega)$, we define the energy functional $E$ and Pohozaev functional $J$ as 


$$
E(u)=\int_{\Omega} \frac{1}{p(x)}|\nabla u|^{p(x)} d x+\int_{\Omega} \frac{1}{q^{2}(x)}|u|^{q(x)} d x-\int_{\Omega} \frac{1}{q(x)}|u|^{q(x)} \log (|u|) d x,
$$

and

$$
J(u)=\int_{\Omega} \frac{1}{p^{*}(x)}|\nabla u|^{p(x)} d x-\int_{\Omega} \frac{1}{q(x)}|u|^{q(x)} \log (|u|) d x .
$$

Let $\mathcal{P}=\left\{u \in W_{0}^{1, p(x)}(\Omega) \backslash\{0\}: J(u)=0\right\}$ be the Pohozaev manifold [10]. Related to the above manifold we have the real number $\Gamma=\inf \{E(u): u \in \mathcal{P}\}$.

To introduce our main results, we first give the definition of weak solutions and blow-up for problem (1).

Definition 1 (Weak solution) $\quad u=u(x, t) \in L^{\infty}\left(0, T ; W_{0}^{1, p(x)}(\Omega)\right) \quad$ with $u_{t} \in L^{2}\left(0, T ; L^{2}(\Omega)\right)$, is said to be a weak solution of problem (1) on $\Omega \times[0, T)$, if it satisfies the initial condition $u(x, 0)=u_{0}(x)$, and the following equality holds

$$
\int_{\Omega} u_{t} w d x+\int_{\Omega} \nabla u_{t} \nabla w d x+\int_{\Omega}|\nabla u|^{p(x)-2} \nabla u \nabla w d x=\int_{\Omega}|u|^{q(x)-2} u \log (|u|) w d x,
$$

for all $\omega \in W_{0}^{1, p(x)}(\Omega)$, and for almost every $t \in(0, T)$.

Definition 2 (Finite time blow-up) Let $u(x, t)$ be a weak solution of problem (1). We say $u(x, t)$ blows up in finite time if the maximal existence time $T$ is finite and $\lim _{t \rightarrow T}\|u\|_{H_{0}^{1}(\Omega)}^{2}=+\infty$.

\section{Preliminaries and Lemmas}

In this section, we will recall some important results of variable exponentials on Lebesgue or Sobolev spaces. For more details on variable exponential Sobolev spaces, please refer to [20-23].

Let $\Omega \subset \mathbb{R}^{N}$ be a smooth bounded domain and $p \in L^{\infty}(\Omega)$ be a measurable function with $p_{+}=$ess $\sup _{x \in \Omega} p(x)$ and $p_{-}=e s s \inf _{x \in \Omega} p(x)$.

The variable exponent Lebesgue space $L^{p(x)}(\Omega)$ is defined by

$$
L^{p(x)}(\Omega)=\left\{u(x) \mid u \text { is measurable in } \Omega \text { and } \int_{\Omega}|u|^{p(x)} d x<\infty\right\}
$$

with the norm

$$
\|u\|_{L^{p(x)}(\Omega)}=\inf \left\{\lambda>0, \int_{\Omega}\left|\frac{u}{\lambda}\right|^{p(x)} d x \leq+\infty\right\} .
$$

The variable exponent Sobolev space $W^{1, p(x)}(\Omega)$ is defined by 


$$
W^{1, p(x)}(\Omega)=\left\{u(x) \in L^{p(x)}(\Omega)|| \nabla u \mid \in L^{p(x)}(\Omega)\right\}
$$

with the norm

$$
\|u\|_{W^{1, p(x)}(\Omega)}=\|u\|_{L^{p(x)}(\Omega)}+\|\nabla u\|_{L^{p(x)}(\Omega)} .
$$

So with these norms, the space $L^{p(x)}(\Omega)$ and $W^{1, p(x)}(\Omega)$ are reflexive and separable Banach spaces. The closure of $C_{0}^{\infty}(\Omega)$ in $W^{1, p(x)}(\Omega)$ is denoted by $W_{0}^{1, p(x)}(\Omega)$, this reflexive Banach space is equipped with the norm $\|u\|_{W_{0}^{1, p(x)}(\Omega)}=\|\nabla u\|_{L^{p(x)}(\Omega)}$.

By virtue of the notion $p_{+}=e s s \sup _{x \in \Omega} p(x), p_{-}=e s s \inf _{x \in \Omega} p(x)$ and $1 \leq p_{-} \leq p(x) \leq p_{+}$a.e. in $x \in \Omega$, we have

$$
\begin{aligned}
\|u\|_{W_{0}^{1, p(x)}(\Omega)}^{p_{-}} & \leq \int_{\Omega}|\nabla u(x)|^{p(x)} d x \leq\|u\|_{W_{0}^{1, p(x)}(\Omega)}^{p_{+}}, \text {if }\|u\|_{W_{0}^{1, p(x)}(\Omega)}<1, \\
\|u\|_{W_{0}^{1, p(x)}(\Omega)}^{p_{+}} & \leq \int_{\Omega}|\nabla u(x)|^{p(x)} d x \leq\|u\|_{W_{0}^{1, p(x)}(\Omega)}^{p_{-}}, \text {if }\|u\|_{W_{0}^{1, p(x)}(\Omega)} \geq 1 .
\end{aligned}
$$

The dual space of $W^{1, p(x)}(\Omega)$ is denoted by $W^{-1, p^{\prime}(x)}(\Omega)$, where $\frac{1}{p(x)}+\frac{1}{p^{\prime}(x)}=1, \forall x \in \bar{\Omega}$.

Related to the properties of logarithms and Lebesgue space $L^{p(x)}(\Omega)$, we have the following logarithm inequality and generalized Hölder-type inequality.

Lemma 1 (Logarithm inequality [10]) The following inequality holds

$$
|\log (s)| \leq \frac{1}{q(x)} s^{q(x)},
$$

for all $s \in[1,+\infty)$ and $q: \bar{\Omega} \rightarrow(0,+\infty)$ is a continuous function satisfy that

$$
0<q^{-}=\min _{x \in \bar{\Omega}} q(x) \leq q(x) \leq q^{+}=\max _{x \in \bar{\Omega}} q(x)<+\infty .
$$

Lemma 2 (Hölder inequality [20]) For any $u \in L^{p(x)}(\Omega)$ and $v \in L^{p^{\prime}(x)}(\Omega)$, then

$$
\left|\int_{\Omega} u v d x\right| \leq\left(\frac{1}{p_{-}}+\frac{1}{p_{-}^{\prime}}\right)\|u\|_{p(x)}\|v\|_{p^{\prime}(x)} .
$$

We conclude this section by recalling the classical interpolation inequality.

Lemma 3 (Interpolation inequality [24]) If $1 \leq p_{0}<p_{\theta}<p_{1} \leq \infty$, then

$$
\|u\|_{p_{\theta}} \leq\|u\|_{p_{0}}^{1-\theta}\|u\|_{p_{1}}^{\theta}
$$

for all $u \in L^{p_{0}}(\Omega) \cap L^{p_{1}}(\Omega)$ with $\theta \in(0,1)$ defined by $\frac{1}{p_{\theta}}=\frac{1-\theta}{p_{0}}+\frac{\theta}{p_{1}}$. 


\section{Main Results}

In this section, we will give the three theorems which are, respectively, related to global existence, local existence and finite time blow-up of solutions involving problem (1). The existence or nonexistence of the global solution is obtained by using the potential well method and the logarithmic inequality. The finite time blow-up phenomenon is obtained by concavity method.

Theorem 1 (Global existence) Let $u_{0} \in W_{0}^{1, p(x)}(\Omega)$ and $E\left(u_{0}\right)<\Gamma, J\left(u_{0}\right)>0$, then the problem (1) has a global weak solution $u(x, t)$ on $\Omega \times[0,+\infty)$.

Proof First we define the potential well associated with the problem (1) as

$$
W=\left\{u \in W_{0}^{1, p(x)}(\Omega): E(u)<\Gamma, J(u)>0\right\} \cup\{0\} .
$$

In the space $W_{0}^{1, p(x)}(\Omega)$, we take a basis $\left\{w_{j}\right\}_{j=1}^{\infty}$ and define the finite dimensional space

$$
V_{m}=\operatorname{Span}\left\{w_{1}, w_{2}, \cdots, w_{m}\right\} .
$$

Let $u_{0 m} \in V_{m}$, then $u_{0 m}=\sum_{j=1}^{m} a_{m i} w_{i} \rightarrow u_{0}$ strongly in $W_{0}^{1, p(x)}(\Omega)$ as $m \rightarrow+\infty$. We look for the approximate solutions of the following form

$$
u_{m}(x, t)=\sum_{j=1}^{m} \alpha_{m j}(t) w_{j}(x), m=1,2, \cdots,
$$

where the coefficients $\alpha_{m j}(t)=\left(u_{m}, w_{j}\right)_{2}$ satisfy the system of ODES

$$
\begin{gathered}
\int_{\Omega} u_{m t} w d x+\int_{\Omega} \nabla u_{m t} \nabla w d x+\int_{\Omega}\left|\nabla u_{m}\right|^{p(x)-2} \nabla u_{m} \nabla w d x \\
=\int_{\Omega}\left|u_{m}\right|^{q(x)-2} u_{m} \log \left(\left|u_{m}\right|\right) w d x, \\
\alpha_{m j}(0)=a_{m j}, j=1,2, \ldots, m .
\end{gathered}
$$

The existence of local solution of system $(13,14)$ is guaranteed by Peano's theorem. Multiplying the equality of (13) by $\alpha_{m_{j}}^{\prime}(t)$, summing for $j$ from 1 to $m$ and integrating with respect to time from 0 to $t$, it yields

$$
\int_{0}^{t}\left\|u_{s m}(s)\right\|_{H_{0}^{1}(\Omega)}^{2} d s+E\left(u_{m}(t)\right)=E\left(u_{0 m}\right), t \in\left[0, t_{0, m}\right] .
$$

From $(12,13,15)$ and the continuity of $E$, we get $E\left(u_{m}(0)\right) \rightarrow E\left(u_{0}\right)$. According to the assumption that $E\left(u_{0}\right)<\Gamma$, we have $E\left(u_{0 m}\right)<\Gamma$ for sufficiently large $m$. Therefore, we obtain 


$$
\int_{0}^{t}\left\|u_{s m}(s)\right\|_{H_{0}^{1}(\Omega)}^{2} d s+E\left(u_{m}(t)\right)<\Gamma, t \in\left[0, t_{0, m}\right],
$$

for sufficiently large $m$. We will show that $t_{0, m}=+\infty$ and

$$
u_{m}(t) \in W, \forall t \geq 0
$$

for sufficiently large $m$. Suppose that (17) does not hold and let $t_{*} \in\left[0, t_{0 m}\right]$ be the smallest time for which $u_{m}\left(t_{*}\right) \notin W$, then by the continuity of the $u_{m}(t)$, we get $u_{m}\left(t_{*}\right) \in \partial W$. Hence, it follows that

$$
E\left(u_{m}\left(t_{*}\right)\right)=\Gamma
$$

or

$$
J\left(u_{m}\left(t_{*}\right)\right)=0 .
$$

If (18) is true, it contradicts with (16). While if (19) is true, then $u_{m}\left(t_{*}\right) \in \mathcal{P}$, $E\left(u_{m}\left(t_{*}\right)\right) \geq \inf _{u \in \mathcal{P}} E(u)=\Gamma$, which also contradicts with (16). Consequently, we have $u_{m}(t) \in W, \forall t \geq 0$.

On the other hand, since $u_{m}(t) \in W$ and (7), it follows that

$$
\begin{aligned}
E\left(u_{m}\right) & \geq \int_{\Omega} \frac{1}{p(x)}\left|\nabla u_{m}\right|^{p(x)} d x-\int_{\Omega} \frac{1}{q(x)}\left|u_{m}\right|^{q(x)} \log \left(\left|u_{m}\right|\right) d x \\
& \geq \int_{\Omega} \frac{1}{p_{+}}\left|\nabla u_{m}\right|^{p(x)} d x-\int_{\Omega} \frac{1}{p^{*}(x)}\left|\nabla u_{m}\right|^{p(x)} d x \\
& \geq \frac{p_{-}^{*}-p_{+}}{p_{-}^{*} p_{+}} \int_{\Omega}\left|\nabla u_{m}\right|^{p(x)} d x .
\end{aligned}
$$

Combining the above inequality with (16), we derive

$$
\int_{0}^{t}\left\|u_{\mathrm{sm}}(m)\right\|_{H_{0}^{1}(\Omega)}^{2} d s<\Gamma \text { and } \int_{\Omega}\left|\nabla u_{m}\right|^{p(x)} d x<\frac{p_{+} p_{-}^{*} \Gamma}{p_{-}^{*}-p_{+}},
$$

from where it follows that $t_{0, m}=+\infty$.

By (20), there exists functions $u, \chi$ and a subsequence of $\left\{u_{m}\right\}_{m=1}^{\infty}$ which we still denote by $\left\{u_{m}\right\}_{m=1}^{\infty}$ such that

$$
\begin{gathered}
u_{m} \rightarrow u \text { weakly star in } L^{\infty}\left((0,+\infty) ; W_{0}^{1, p(x)}(\Omega)\right) \\
u_{m t} \rightarrow u_{t} \text { weakly star in } L^{2}\left((0,+\infty) ; H_{0}^{1}(\Omega)\right) \\
-\operatorname{div}\left(\left|\nabla u_{m}\right|^{p(x)-2} \nabla u_{m}\right) \rightarrow \chi(t) \text { weakly star in } L^{\infty}\left((0,+\infty) ; W_{0}^{-1, p^{\prime}(x)}(\Omega)\right) .
\end{gathered}
$$

By $(21,22)$ and Aubin-Lions-Simon Lemma ([25], Corollary 4), we get 


$$
u_{m} \rightarrow u \text { strong in } C\left((0,+\infty), L^{r(x)}(\Omega)\right), \quad \forall r(x) \in\left[2, p^{*}(x)\right), x \in \bar{\Omega},
$$

thus

$$
u_{m} \rightarrow u \text { a.e. } x \in \Omega, \forall t>0
$$

This implies

$$
\left|u_{m}\right|^{q(x)-2} u_{m} \log \left(\left|u_{m}\right|\right) \rightarrow|u|^{q(x)-2} u \log (|u|) \text { a.e. } x \in \Omega, \forall t>0 .
$$

On the other hand, by a direct calculation, we have

$$
\begin{aligned}
& \left.\left.\int_{\Omega}|| u_{m}\right|^{q(x)} \log \left(\left|u_{m}\right|\right)\right|^{\frac{q_{+}}{q_{+}-1}} d x \\
\leq & \left.\left.\int_{x \in \Omega,\left|u_{m}\right| \geq 1}|| u_{m}\right|^{q(x)-1} \log \left(\left|u_{m}\right|\right)\right|^{\frac{q_{+}}{q_{+}-1}} d x \\
& +\left.\left.\int_{x \in \Omega,\left|u_{m}\right| \leq 1}|| u_{m}\right|^{q(x)-1} \log \left(\left|u_{m}\right|\right)\right|^{\frac{q_{+}}{q_{+}-1}} d x \\
\leq & \left.\left.\int_{x \in \Omega,\left|u_{m}\right| \geq 1}|| u_{m}\right|^{q(x)-1} \log \left(\left|u_{m}\right|\right)\right|^{\frac{q_{+}}{q_{+}-1}} d x+c|\Omega| \\
\leq & \left.\left.\int_{x \in \Omega,\left|u_{m}\right| \geq 1}|| u_{m}\right|^{q(x)}\right|^{\frac{q_{+}}{q_{+}-1}} d x+c|\Omega| \\
\leq & c|\Omega|+c|\Omega| \cdot|| u_{m}||_{L^{p^{*}}(\Omega)} \\
\leq & c .
\end{aligned}
$$

Hence, by Lion's Lemma (see [25], Lemma 1.3), it yields

$$
\left|u_{m}\right|^{q(x)-2} u_{m} \log \left(\left|u_{m}\right|\right) \longrightarrow|u|^{q(x)-2} u \log \left(\left|u_{m}\right|\right),
$$

weakly star in $L^{\infty}\left((0,+\infty) ; L^{q^{\prime}(x)}(\Omega)\right)$.

By $(21,22,23)$ and (27), passing to the limit in (10) and (12) as $m \rightarrow \infty$, we can show that $u$ satisfies the initial condition $u(0)=u_{0}$ and

$$
\int_{\Omega} u_{t} w d x+\int_{\Omega} \nabla u_{t} \nabla w d x+<\chi(t), w>=\int_{\Omega}|u|^{q(x)-2} u \log (|u|) w d x .
$$

The next step is to prove that

$$
\chi(t)=-\operatorname{div}\left(|\nabla u|^{p(x)-2} \nabla u\right) .
$$

Let $A u=-\operatorname{div}\left(|\nabla u|^{p(x)-2} \nabla u\right)$, we observe $A: W_{0}^{1, p(x)}(\Omega) \rightarrow W_{0}^{-1, p^{\prime}(x)}(\Omega)$ is bounded, monotone and hemicontinuous operator (see [21]). In addition,

$$
\langle A u, u\rangle=\int_{\Omega}|\nabla u|^{p(x)} d x, \forall u \in W_{0}^{1, p(x)}(\Omega) .
$$

From (27), we have 


$$
\int_{0}^{T} \int_{\Omega}\left|u_{m}\right|^{q(x)-2} u_{m} \log \left(\left|u_{m}\right|\right) \omega d x d t \rightarrow \int_{0}^{T} \int_{\Omega}|u|^{q(x)-2} u \log (|u|) \omega d x d t,
$$

for any $\omega \in L^{q_{+}}(\Omega)$. We observe that

$$
\begin{aligned}
& \left.\left|\int_{\Omega}\right| u_{m}\right|^{q(x)} \log \left(\left|u_{m}\right|\right)-|u|^{q(x)} \log (|u|) d x \mid \\
\leq & \left.\left|\int_{\Omega}\left(u_{m}-u\right)\right| u_{m}\right|^{q(x)-2} u_{m} \log \left(\left|u_{m}\right|\right) d x \mid \\
& +\left|\int_{\Omega} u\left(\left|u_{m}\right|^{q(x)-2} u_{m} \log \left(\left|u_{m}\right|\right)-|u|^{q(x)-2} u \log (|u|)\right) d x\right| .
\end{aligned}
$$

From (30), we have

$$
\left|\int_{\Omega} u\left(\left|u_{m}\right|^{q(x)-2} u_{m} \log \left(\left|u_{m}\right|\right)-|u|^{q(x)-2} u \log (|u|)\right) d x\right| \rightarrow 0,
$$

as $m \rightarrow \infty$.

Using the Hölder inequality, we get

$$
\left.\left|\int_{\Omega}\left(u_{m}-u\right)\right| u_{m}\right|^{q(x)-2} u_{m} \log \left(\left|u_{m}\right|\right) d x\left|\leq\left\|u_{m}-u\right\|_{q_{+}}\left\|\left|u_{m}\right|^{q(x)} u_{m} \log \left(\left|u_{m}\right|\right)\right\|_{\frac{q_{+}}{q_{+}-1}} .\right.
$$

From (24) we have

$$
\left.\left|\int_{\Omega}\left(u_{m}-u\right)\right| u_{m}\right|^{q(x)-2} u_{m} \log \left(\left|u_{m}\right|\right) d x \mid \rightarrow 0
$$

as $m \rightarrow \infty$.

This combined with (31) implies that

$$
\int_{\Omega}\left|u_{m}\right|^{q(x)} \log \left(\left|u_{m}\right|\right) d x \rightarrow \int_{\Omega}|u|^{q(x)} \log (|u|) d x .
$$

Integrating (28) with respect to time from 0 to $T$, we can obtain

$$
\begin{gathered}
\int_{0}^{T} \int_{\Omega} u_{t} w d x d t+\int_{0}^{T} \int_{\Omega} \nabla u_{t} \nabla w d x d t+\int_{0}^{T}\langle\chi(t), w\rangle d t \\
=\int_{0}^{T} \int_{\Omega}|u|^{q(x)-2} u \log (|u|) w d x d t
\end{gathered}
$$

Taking $u=w$ in (33), we obtain 


$$
\begin{aligned}
& \int_{0}^{T}\langle\chi(t), u\rangle d t \\
= & \int_{0}^{T} \int_{\Omega}|u|^{q(x)} \log (|u|) d x d t-\frac{1}{2}\|u(T)\|_{H_{0}^{1}(\Omega)}^{2}+\frac{1}{2}\|u(0)\|_{H_{0}^{1}(\Omega)}^{2} .
\end{aligned}
$$

Multiplying the equality of (13) by $\alpha_{m_{j}}(t)$, summing over $j$ form 1 to $m$ and integrating over $(0, T)$, it yields

$$
\begin{aligned}
& \frac{1}{2}\left\|u_{m}(T)\right\|_{H_{0}^{1}(\Omega)}^{2}-\frac{1}{2}\left\|u_{m}(0)\right\|_{H_{0}^{1}(\Omega)}^{2}+\int_{0}^{T}\left\langle A u_{m}, u_{m}\right\rangle d t \\
= & \int_{0}^{T} \int_{\Omega}\left|u_{m}\right|^{q(x)} \log \left(\left|u_{m}\right|\right) d x d t .
\end{aligned}
$$

From (24) and (32), we deduce that

$$
\begin{aligned}
& \lim _{m \rightarrow \infty} \sup \int_{0}^{T}\left\langle A u_{m}, u_{m}\right\rangle d t \\
= & \int_{0}^{T} \int_{\Omega}|u|^{q(x)} \log (|u|) d x d t-\frac{1}{2}\|u(T)\|_{H_{0}^{1}(\Omega)}^{2}+\frac{1}{2}\|u(0)\|_{H_{0}^{1}(\Omega)}^{2} .
\end{aligned}
$$

Combing (34) and (36), we obtain

$$
\lim _{m \rightarrow \infty} \sup \int_{0}^{T}\left\langle A u_{m}, u_{m}\right\rangle d t=\int_{0}^{T}\langle\chi(t), u\rangle d t .
$$

Finally, by (37) and ([26] Lemma 3.2.2) we deduce that (29) holds, which implies

$$
\int_{\Omega} u_{t} w d x+\int_{\Omega} \nabla u_{t} \nabla w d x+\int_{\Omega}|\nabla u|^{p(x)-2} \nabla u \nabla w d x=\int_{\Omega}|u|^{q(x)-2} u \log (|u|) w d x
$$

for all $\left.w \in W_{0}^{1, p(x)}(\Omega)\right)$ and a.e. $t \in[0, \infty)$.

In view of Definition 1 and the above discussions, we get the global existence of the solution of problem (1). The proof of Theorem 1 is complete.

Theorem 2 (Local existence) Let $u_{0} \in W_{0}^{1, p(x)}(\Omega)$ and assume $2<q_{-}<q(x)<q_{+}<\frac{N p_{-}}{N-p_{-}}, \forall x \in \bar{\Omega}$, then the problem (1) admit a local weak solution. Moreover, $u$ satisfy the energy inequality

$$
\int_{0}^{t}\left\|u_{s}(s)\right\|_{H_{0}^{1}(x)}^{2} d s+E(u(t)) \leq E\left(u_{0}\right) \text { a.e. } t \in\left[0, T_{*}\right]
$$

Proof Similar to the proof of Theorem 1, by means of Galerkin method, we consider the approximate solution of problem (1) as $u_{m}(x, t)=\sum_{j=1}^{m} \beta_{m j}(t) w_{j}(x)$, which satisfies the following equations 


$$
\begin{gathered}
\int_{\Omega} u_{m t} w_{i} d x+\int_{\Omega} \nabla u_{m t} \nabla w_{i} d x+\int_{\Omega}\left|\nabla u_{m}\right|^{p(x)-2} \nabla u_{m} \nabla w_{i} d x \\
=\int_{\Omega}\left|u_{m}\right|^{q(x)-2} u_{m} \log \left|u_{m}\right| w_{i} d x, \\
u_{m}(0)=u_{0 m} .
\end{gathered}
$$

Due to $u_{0} \in W_{0}^{1, p(x)}(\Omega)$, then it exists $\left\{b_{m j}, j=1,2, \cdots, m\right\}$ such that

$$
u_{m}(0)=\sum_{j=1}^{m} b_{m j} w_{j} \rightarrow u_{0} \text { strongly in } W_{0}^{1, p(x)}(\Omega) .
$$

The existence of local solution of system (40) (41) is guaranteed by Peano's theorem.

Multiplying the equality of (40) by $\beta_{m j}(t)$ and summing over $j$ from 1 to $m$, we obtain

$$
\frac{1}{2} \frac{d}{d t}\left\|u_{m}\right\|_{\mathrm{H}_{0}^{1}(\Omega)}^{2}+\int_{\Omega}\left|\nabla u_{m}\right|^{p(x)} d x=\int_{\Omega}\left|u_{m}\right|^{q(x)} \log \left(\left|u_{m}\right|\right) d x .
$$

By virtue of Lemma 1, we have

$$
\begin{aligned}
& \int_{\Omega}\left|u_{m}\right|^{q(x)} \log \left(\left|u_{m}\right|\right) d x \\
\leq & \int_{\left\{x \in \Omega|| u_{m} \mid>1\right\}}\left|u_{m}\right|^{q(x)} \log \left(\left|u_{m}\right|\right) d x \\
\leq & \int_{\left\{x \in \Omega|| u_{m} \mid>1\right\}}\left|u_{m}\right|^{q_{+}} \log \left(\left|u_{m}\right|\right) d x \\
\leq & \frac{1}{\mu} \int_{\Omega}\left|u_{m}\right|^{q_{+}+\mu} d x=\frac{1}{\mu}\left\|u_{m}(t)\right\|_{q_{+}+\mu}^{q_{+}+\mu} .
\end{aligned}
$$

Choosing $\mu$ such that $2<p_{-}<\mu<\frac{N p_{-}}{N-p_{-}}-q_{+}$, we have

$$
\int_{\Omega}\left|u_{m}\right|^{q(x)} \log \left(\left|u_{m}\right|\right) d x \leq\left\|u_{m}\right\|_{2}^{(1-\theta)\left(q_{+}+\mu\right)}\left\|u_{m}\right\|_{\frac{N p_{-}}{N-p_{-}}}^{\theta\left(q_{+}+\mu\right)},
$$

where $\theta \in(0,1)$ and $\frac{1}{\mu+q_{+} N p_{-}}=\frac{\theta\left(N-p_{-}\right)}{N p_{-}}+\frac{1-\theta}{2}$.

Due to $L^{p *(x)}(\Omega) \hookrightarrow L^{\frac{+v p_{-}}{N-p_{-}}}(\Omega)$ and $W^{1, p(x)}(\Omega) \hookrightarrow L^{p *(x)}(\Omega)$, we have

$$
\left\|u_{m}\right\|_{\frac{N p_{-}}{N-p_{-}}} \leq c\left\|u_{m}\right\|_{W_{0}^{1, p(x)}(\Omega)} .
$$

Combing the above inequality with (44), we obtain

$$
\int_{\Omega}\left|u_{m}\right|^{q(x)} \log \left(\left|u_{m}\right|\right) \leq c|| u_{m}\left\|_{2}^{(1-\theta)\left(q_{+} \mu\right)}\right\| u_{m} \|_{W_{0}^{1, p(x)}(\Omega)}^{\theta\left(q_{+}+\mu\right)} .
$$

By virtue of $H_{0}^{1}(\Omega) \hookrightarrow L^{2}(\Omega)$, it yields 


$$
\int_{\Omega}\left|u_{m}\right|^{q(x)} \log \left(\left|u_{m}\right|\right) d x \leq c|| u_{m}\left\|_{H_{0}^{1}(\Omega)}^{(1-\theta)\left(q_{+} \mu\right)}\right\| u_{m} \|_{W_{0}^{1, p(x)}(\Omega)}^{\theta\left(q_{+}+\mu\right)} .
$$

We discuss the proof into two cases as follows.

Case 1 Suppose $\left\|u_{m}\right\| \geq 1$, then

$$
\int_{\Omega}\left|u_{m}\right|^{q(x)} \log \left(\left|u_{m}\right|\right) d x \leq c|| u_{m} \|_{H_{0}^{1}(\Omega)}^{(1-\theta)\left(q_{+}+\mu\right)}\left(\int_{\Omega}\left|\nabla u_{m}\right|^{p(x)} d x\right)^{\frac{\theta\left(q_{+}+\mu\right)}{p_{-}}} .
$$

Due to $\frac{1}{\mu+q_{+}}=\frac{\theta\left(N-p_{-}\right)}{N p_{-}}+\frac{1-\theta}{2}$, we get

$$
1=\frac{\theta\left(N-p_{-}\right)\left(\mu+q_{+}\right)}{N p_{-}}+\frac{(1-\theta)\left(\mu+q_{+}\right)}{2}>\frac{\theta\left(N-p_{-}\right)\left(\mu+q_{+}\right)}{N p_{-}} .
$$

Setting $\frac{N-p_{-}}{N p_{-}}<\frac{1}{2}$, we obtain

$$
\frac{p_{-}}{\theta\left(q_{+}+\mu\right)}>1 \text {. }
$$

By Young's inequality and (46), it follows that

$$
\int_{\Omega}\left|u_{m}\right|^{q(x)} \log \left(\left|u_{m}\right|\right) d x \leq c\left(\left\|u_{m}\right\|_{H_{0}^{1}(\Omega)}^{2}\right)^{\frac{(1-\theta)\left(q_{+}+\mu\right) p_{-}}{2 p_{-}-2 \theta\left(q_{+} \mu\right)}}+\varepsilon \int_{\Omega}\left|\nabla u_{m}\right|^{p(x)} d x .
$$

Case 2 Suppose $\left\|u_{m}\right\| \leq 1$, then

$$
\int_{\Omega}\left|u_{m}\right|^{q(x)} \log \left(\left|u_{m}\right|\right) d x \leq c\left(\left\|u_{m}\right\|_{H_{0}^{1}(\Omega)}^{2}\right)^{\frac{(1-\theta)\left(q_{+}+\mu\right)}{2}} .
$$

Due to $p_{-}-\theta\left(q_{+}+\mu\right)$ and $\frac{p_{-}}{p_{-}-\theta\left(q_{+}+\mu\right)}>1$, it follows that

$$
\int_{\Omega}\left|u_{m}\right|^{q(x)} \log \left(\left|u_{m}\right|\right) d x \leq c+\left(\|\left. u_{m}\right|_{H_{0}^{1}(\Omega)} ^{2}\right)^{\frac{(1-\theta)\left(q_{+}+\mu\right) p_{-}}{2 p_{-}-2 \theta\left(q_{+}+\mu\right)}} .
$$

By (47) and (49), we obtain

$$
\int_{\Omega}\left|u_{m}\right|^{q(x)} \log \left(\left|u_{m}\right|\right) d x \leq c\left(\left\|u_{m}\right\|_{H_{0}^{1}(\Omega)}^{2}\right)^{\frac{(1-\theta)\left(q_{+}+\mu\right) p_{-}}{2 p_{-}-2 \theta\left(q_{+} \mu\right)}}+\varepsilon \int_{\Omega}\left|\nabla u_{m}\right|^{p(x)} d x .
$$

Choosing $\alpha=\frac{(1-\theta)\left(q_{+}+\mu\right) p_{-}}{2 p_{-}-2 \theta\left(q_{+} \mu\right)}$ and using $\frac{1}{\mu+q_{+}}=\frac{\theta\left(N-p_{-}\right)}{N p_{-}}+\frac{1-\theta}{2}$, we derive that

$$
\alpha-1=\frac{\theta p_{-}\left(\mu+q_{+}\right)}{N\left[p_{-}-\theta\left(q_{+}+\mu\right)\right]}>0 .
$$

Setting $\varepsilon \in(0,1)$, we have

$$
\frac{1}{2} \frac{d}{d t}\left\|u_{m}\right\|_{H_{0}^{1}(\Omega)}^{2}+\int_{\Omega}\left\|\nabla u_{m}\right\|^{p(x)} d x \leq c\left(\left\|u_{m}\right\|_{H_{0}^{1}(\Omega)}\right)^{\alpha}+\varepsilon \int_{\Omega}\left|\nabla u_{m}\right|^{p(x)} d x .
$$

Let $S_{m}(t)=\left\|u_{m}\right\|_{H_{0}^{1}(\Omega)}^{2}$, we obtain 


$$
S_{m}^{\prime}(t)=c\left(S_{m}(t)\right)^{\alpha}
$$

Integrating inequality (51) with respect to time from 0 to $t$, we get

$$
\left(S_{m}(t)\right)^{1-\alpha} \leq c(1-\alpha) t+\left(S_{m}(0)\right)^{1-\alpha} .
$$

Since $\quad\left(S_{m}(t)\right)^{1-\alpha}>0, \quad$ it $\quad$ yields $\quad t \leq \frac{S_{m}^{1-\alpha}(0)}{c(\alpha-1)}=T_{*} \quad$ and $S_{m}(t)=\left\|u_{m}\right\|_{H_{0}^{1}(\Omega)}^{2}<c, \forall t \in\left[0, T_{*}\right]$.

Multiplying the equality of (40) by $\beta_{m j}^{\prime}(t)$ and summing over $j$ from 1 to $m$, and integrating over $(0, \mathrm{t})$ yields

$$
\int_{0}^{t}\left\|u_{m s}(s)\right\|_{H_{0}^{1}(\Omega)}^{2} d s+E\left(u_{m}(t)\right)=E\left(u_{m}(0)\right) .
$$

By the continuity of the functional $E$ and (41), there exists a constant $c>0$ satisfying

$$
E\left(u_{0 m}\right) \leq c,
$$

for any positive integer $m$.

On the other hand,

$$
\begin{aligned}
E\left(u_{m}\right) & =\int_{\Omega} \frac{1}{p(x)}\left|\nabla u_{m}\right|^{p(x)} d x+\int_{\Omega} \frac{1}{q^{2}(x)}\left|u_{m}\right|^{q(x)} d x-\int_{\Omega} \frac{1}{q(x)}\left|u_{m}\right| \log \left|u_{m}\right| d x \\
& \geq \int_{\Omega} \frac{1}{p(x)}\left|\nabla u_{m}\right|^{p(x)} d x-\int_{\Omega} \frac{1}{q(x)}\left|u_{m}\right|^{q(x)} \log \left|u_{m}\right| d x \\
& \geq \frac{1}{p_{-}} \int_{\Omega}\left|\nabla u_{m}\right|^{p(x)} d x-\frac{1}{q_{+}} \int_{\Omega}\left|u_{m}\right|^{q(x)} \log \left|u_{m}\right| d x \\
& \geq \frac{1}{p_{-}} \int_{\Omega}\left|\nabla u_{m}\right|^{p(x)} d x-\frac{\varepsilon}{q_{+}} \int_{\Omega}\left|\nabla u_{m}\right|^{p(x)} d x-\frac{c}{q_{+}}\left(|| u_{m}||_{H_{0}^{1}(\Omega)}^{2}\right)^{\alpha} \\
& =\left(\frac{1}{p_{-}}-\frac{\varepsilon}{q_{+}}\right) \int_{\Omega}\left|\nabla u_{m}\right|^{p(x)} d x-\frac{c}{q_{+}}\left(\left.|| u_{m}\right|_{H_{0}^{1}(\Omega)} ^{2}\right)^{\alpha},
\end{aligned}
$$

thus

$$
\left(\frac{1}{p_{-}}-\frac{\varepsilon}{q_{+}}\right) \int_{\Omega}\left|\nabla u_{m}\right|^{p(x)} d x-\frac{c}{q_{+}}\left(\left\|u_{m}\right\|_{H_{0}^{1}(\Omega)}^{2}\right)^{\alpha}<c .
$$

By $(53,54,55)$ and $\left\|u_{m}\right\|_{H_{0}^{1}(\Omega)}^{2}<c$, there exists functions $u, \chi$ and a subsequence of $\left\{u_{m}\right\}_{m=1}^{\infty}$ which we still denote by $\left\{u_{m}\right\}_{m=1}^{\infty}$ such that

$$
\begin{gathered}
u_{m} \rightarrow u \text { weakly star in } L^{\infty}\left(\left[0, T_{*}\right] ; W_{0}^{1, p(x)}(\Omega)\right), \\
u_{m t} \rightarrow u_{t} \text { weakly star in } L^{2}\left(\left[0, T_{*}\right] ; H_{0}^{1}(\Omega)\right),
\end{gathered}
$$




$$
-\operatorname{div}\left(\left|\nabla u_{m}\right|^{P(x)-2} \nabla u_{m}\right) \rightarrow \chi(t) \text { weakly star in } L^{\infty}\left(\left[0, T_{*}\right] ; W_{0}^{-1, p^{\prime}(x)}(\Omega)\right)
$$

The remaining proof of the existence of local solutions is similar as described in Theorem 1 , then it yields

$$
\int_{\Omega} u_{t} w d x+\int_{\Omega} \nabla u_{t} \nabla w d x+\int_{\Omega}|\nabla u|^{p(x)-2} \nabla u \nabla w d x=\int_{\Omega}|u|^{q(x)-2} u \log |u| w d x,
$$

for all $\omega \in W_{0}^{1, p(x)}(\Omega)$, a.e. in $\left[0, T_{*}\right]$.

In view of the Definition 1, we get the local existence of the solution of problem (1). Now, let's prove that the solution satisfies the energy inequality. By (53), we get

$$
\int_{0}^{T_{*}} \theta(t) d t \int_{0}^{t}\left\|u_{m s}(s)\right\|_{H_{0}^{1}(\Omega)}^{2} d s+\int_{0}^{T_{*}} E\left(u_{m}(t)\right) \theta(t) d t=\int_{0}^{T_{*}} E\left(u_{m}(0)\right) \theta(t) d t .
$$

Due to the second term of the left-hand side $\int_{0}^{T_{*}} E\left(u_{m}(t)\right) \theta(t) d t$ is lower semi-continuous with respect to the weak topology of $W_{0}^{1, p(x)}(\Omega)$, then it follows that

$$
\int_{0}^{T_{*}} E(u(t)) \theta(t) d t \leq \underline{\lim _{n \rightarrow \infty}} \int_{0}^{T_{*}} E\left(u_{m}(t)\right) \theta(t) d t .
$$

By (59) and (60) and letting $m \rightarrow+\infty$, we have

$$
\int_{0}^{T_{*}} \theta(t) d t \int_{0}^{1}\left\|u_{s}(s)\right\|_{H_{0}^{1}(\Omega)}^{2} d s+\int_{0}^{T_{*}} E(u(t)) \theta(t) d t \leq \int_{0}^{T_{*}} E(u(0)) \theta(t) d t .
$$

Since $\theta(t)$ is arbitrary, we get

$$
\int_{0}^{t}\left\|u_{s}(s)\right\|_{H_{0}^{1}(x)}^{2} d s+E(u(t)) \leq E\left(u_{0}\right) \text {, a.e. } t \in\left[0, T_{*}\right] .
$$

The proof of Theorem 2 is complete.

Theorem 3 (Blow-up) Let $u_{0} \in W_{0}^{1, p(x)}(\Omega)$ and $2<q_{-}<q(x)<q_{+}<\frac{N p_{-}}{N-p_{-}}$. If $E\left(u_{0}\right)<0, p_{+}<q(x)=q$, the local weak solution of problem (1) blows up at finite time.

\section{Proof Defining}

$$
\Gamma(t)=\frac{1}{2} \int_{0}^{t}\|u(s)\|_{H_{0}^{1}(\Omega)}^{2} d s+\frac{T-t}{2}\left\|u_{0}\right\|_{H_{0}^{1}(\Omega)}^{2}, 0 \leq t<T,
$$

and then taking the second derivative of $\Gamma(t)$, we get

$$
\Gamma^{\prime \prime}(t)=\left(u_{t}(t), u(t)\right)+\left(\nabla u_{t}(t), \nabla u(t)\right) .
$$

By (38) and taking $\omega=\mu$, we obtain 


$$
\begin{aligned}
\Gamma^{\prime \prime}(t) & =-\int_{\Omega}|\nabla u|^{p(x)} d x+\int_{\Omega}|u|^{q(x)} \log (|u|) d x \\
& \geq-\int_{\Omega}|\nabla u|^{p(x)} d x+\int_{\Omega}|u|^{q(x)} \log (|u|) d x+\int_{\Omega}\left(\frac{q}{p_{+}}-\frac{q}{p(x)}\right)|\nabla u|^{p(x)} d x \\
& =\left.\left(\frac{q}{p_{+}}-1\right) \int_{\Omega}|\nabla u|^{p(x)}\left|d x+\frac{1}{q} \int_{\Omega}\right| u\right|^{q} d x-q E(u(t)) .
\end{aligned}
$$

It implies $\Gamma^{\prime \prime}(t) \geq-q E(u(t))$. Due to the energy equality (61), we have

$$
\Gamma^{\prime \prime}(t)-\beta \geq-q E((u(t)))+q\left(E\left(u_{0}\right)\right) \geq q \int_{0}^{t}\left\|u_{s}(s)\right\|_{H_{0}^{1}(\Omega)}^{2} d s,
$$

where $\beta=-q E\left(u_{0}\right)$.

By $\Gamma^{\prime}(t)=\int_{0}^{t}\left(u_{t}(s), u(s)\right)_{H_{0}^{1}(\Omega)} d s$ and Hölder inequality, we obtain

$$
\begin{aligned}
\left(\Gamma^{\prime}(t)\right)^{2} & \leq \int_{0}^{t}\left\|u_{s}(s)\right\|_{H_{0}^{1}(\Omega)}^{2} d s \int_{0}^{t}\|u(s)\|_{H_{0}^{1}(\Omega)}^{2} d s \\
& \leq 2 \int_{0}^{t}\left\|u_{s}(s)\right\|_{H_{0}^{1}(\Omega)}^{2} d s\left(\frac{1}{2} \int_{0}^{t}\|u(s)\|_{H_{0}^{1}(\Omega)}^{2} d s+\frac{T-t}{2}\left\|u_{0}\right\|_{H_{0}^{1}(\Omega)}^{2}\right) \\
& =2 \int_{0}^{t}\left\|u_{s}(s)\right\|_{H_{0}^{1}(\Omega)}^{2} d s(\Gamma(t)),
\end{aligned}
$$

then $\Gamma(t) \Gamma^{\prime \prime}(t)-\frac{q}{2}\left(\Gamma^{\prime}(t)\right)^{2} \geq \Gamma(t) \beta>0, t \in[0, T]$.

Let $y(t)=(\Gamma(t))^{-\frac{q-2}{2}}$, then

$$
y^{\prime}(t)=-\frac{q-2}{2} \Gamma^{-\frac{q}{2}}(t) \Gamma^{\prime}(t)
$$

and

$$
\begin{aligned}
y^{\prime}(t) & =-\frac{q-2}{2} \Gamma^{-\frac{q}{2}}(t) \Gamma^{\prime \prime}(t)+\frac{q}{2}\left(\frac{q-2}{2}\right)(\Gamma(t))^{-\frac{q+2}{2}}\left(\Gamma^{\prime}(t)\right)^{2} \\
& =\Gamma^{-\frac{q}{2}}(t)\left(\frac{2-q}{2} \Gamma^{\prime \prime}(t)+\frac{q(q-2)}{4}\left(\Gamma^{\prime}(t)\right)^{2} \Gamma^{-1}(t)\right) \\
& \leq \Gamma^{-\frac{q}{2}}(t)\left(\frac{2-q}{2} \Gamma^{\prime \prime}(t)+\frac{(q-2)}{2}\left(\Gamma^{\prime \prime}(t)\right)-\beta\right) \\
& =\Gamma^{-\frac{q}{2}}(t)\left(-\frac{q-2}{2} \beta\right)<0 .
\end{aligned}
$$

Since $y^{\prime \prime}(t)<0, y(t)$ is a concave function and $y^{\prime}(t) \leq y^{\prime}(0)=0,0 \leq t<T$. And due to $y^{\prime}(t)<0$ and $y^{\prime \prime}(t)<0,0 \leq t<T$, it implies as long as $T$ is large enough, there exists $T_{*} \in[0, T), \lim _{t \rightarrow T_{*}^{-}} y(t)=0$. Consequently, we obtain $\lim _{t \rightarrow T_{*}^{-}}\|u(s)\|_{H_{0}^{1}(\Omega)}^{2}=+\infty$.

In view of the Definition 2, we get the blow-up of the solution of problem (1). The proof of Theorem 3 is complete. 


\section{Conclusion}

In this paper, by virtue of the potential well method and logarithmic inequalities, we obtain the global existence of solutions of problem (1). What's more, the finite time blow-up phenomenon is obtained by concavity method. The first attempt to study the properties of the solutions for such kind of equations will enrich the research of mathematical physics equations.

Author Contributions All authors completed the paper together. All authors read and approved the final manuscript.

Funding This research was supported by the project of Guizhou province science and technology plan under (No. Qiankehe foundation-ZK[2021]YIBAN317), and by the project of Guizhou Minzu University under (No. GZMU[2019]YB04).

Availability of data and materials Not applicable.

Code availability Not applicable.

\section{Declarations}

Conflict of interest The authors declare that they have no competing interests.

Ethics approval Not applicable.

Consent to participate Not applicable.

Consent for publication Not applicable.

Open Access This article is licensed under a Creative Commons Attribution 4.0 International License, which permits use, sharing, adaptation, distribution and reproduction in any medium or format, as long as you give appropriate credit to the original author(s) and the source, provide a link to the Creative Commons licence, and indicate if changes were made. The images or other third party material in this article are included in the article's Creative Commons licence, unless indicated otherwise in a credit line to the material. If material is not included in the article's Creative Commons licence and your intended use is not permitted by statutory regulation or exceeds the permitted use, you will need to obtain permission directly from the copyright holder. To view a copy of this licence, visit http://creativecommons.org/licen ses/by/4.0/.

\section{References}

1. Barenblatt, G.I., Zheltov, I.P., Kochina, I.N.: Basic concepts in the theory of seepage of homogeneous liquids in fissured rocks [strata]. J. Appl. Math. Mech. 24(5), 1286-1303 (1960)

2. Chen, P.J., Gurtin, M.E.: On a theory of heat conduction involving two temperatures. Z. Angew. Math. Phys. 19(4), 614-627 (1968)

3. Korpusov, M.O., Sveshnikov, A.G.: Three-dimensional nonlinear evolutionary pseudoparabolic equations in mathematical physics. Zh. Vychisl. Mat. Fiz. 43(12), 1835-1869 (2003)

4. Halsey, T.C.: Electrorheological fluids. Science 258(5083), 761-766 (1992)

5. Rajagopal, K., Ružička, M.: Mathematical modeling of electrorheological materials. Continuum Mech. Therm. 13, 59-78 (2001) 
6. Chen, H., Luo, P., Liu, G.: Global solution and blow-up of a semilinear heat equation with logarithmic nonlinearity. J. Math. Anal. Appl. 422(1), 84-98 (2015)

7. Peng, J., Zhou, J.: Global existence and blow-up of solutions to a semilinear heat equation with logarithmic nonlinearity. Appl. Anal. 100(13), 2804-2824 (2021)

8. Nhan, L.C., Truong, L.X.: Global solution and blow-up for a class of pseudo $p$-Laplacian evolution equations with logarithmic nonlinearity. Comput. Math. Appl. 73(9), 2076-2091 (2017)

9. He, Y.J., Gao, H.H., Wang, H.: Blow-up and decay for a class of pseudo-parabolic p-Laplacian equation with logarithmic nonlinearity. Comput. Math. Appl. 75(2), 459-469 (2018)

10. Boudjeriou, T.: On the diffusion $p(x)$-Laplacian with logarithmic nonlinearity. J. Elliptic Parabol. Equ. 6, 773-794 (2020)

11. Lourêdo, A.T., Milla, M.M., Clark, M.R.: Variable exponent perturbation of a parabolic equation with $p(x)$-Laplacian. Electron. J. Qual. Theo. 60, 1-14 (2019)

12. Alves, C.O., Ferreira, M.C.: Existence of solutions for a class of $p(x)$-Laplacian equations involving a concave-convex nonlinearity with critical growth in $\mathbb{R}^{N}$. Toplo. Method. Nonl. An. 45(2), 399-422 (2015)

13. Guo, B., Li, Y.J., Gao, W.J.: Singular phenomena of solutions for nonlinear diffusion equations involving $p(x)$-Laplace operator and nonlinear sources. Z. Angew. Math. Phys. 66(3), 989-1005 (2015)

14. Shangerganesh, L., Gurusamy, A., Balachandran, K.: Weak solutions for nonlinear parabolic equations with variable exponents. Commun. Math. Stat. 25(1), 55-70 (2017)

15. Tsutsumi, M.: On solutions of semilinear differential equations in Hilbert space. Math. Japon. 17, 173-193 (1972)

16. Levine, H.A.: Some nonexistence and instability theorems for solutions of formally parabolic equations of the form $P u_{t}=-A u+F(u)$. Arch. Ration. Mech. Anal. 51(5), 371-386 (1973)

17. Payne, L.E., Sattinger, D.H.: Saddle points and instability of nonlinear hyperbolic equations. Israel J. Math. 22(3), 273-303 (1975)

18. Liu, Y.C.: On potential wells and vacuum isolating of solutions for semilinear wave equations. J. Differ. Equ. 192(1), 155-169 (2003)

19. Liu, Y.C., Zhao, J.S.: On potential wells and applications to semilinear hyperbolic equations and parabolic equations. Nonlinear Anal. 64(12), 2665-2687 (2006)

20. Musielak, J.: Orlicz spaces and modular spaces. Springer, Berlin (1983)

21. Diening, L., Harjulehto, P., Hästö, P., Ruzicka, M.: Lebesgue and Sobolev spaces with variable exponents. Springer, Berlin (2017)

22. Radulescu, V.D., Repovs, D.D.: Partial differential equations with variable exponents: variational methods and qualitative analysis. CRC Press, New York (2015)

23. Kováčik, O., Rákosník, J.: On spaces $L^{p(x)}$ and $W^{k, p(x)}$. Czech. Math. J. 41(4), 592-618 (1991)

24. Tartar, L.: An introduction to Sobolev spaces and interpolation spaces. Springer, Berlin (2007)

25. Simon, J.: Compact sets in the space $L^{p}(O, T ; B)$. Ann. Mat. Pur. Appl. 146(1), 65-96 (1986)

26. Zheng, S.M.: Nonlinear Evolution Equations. CRC Press, New York (2004) 